\title{
Electronic properties of twisted bilayer nanoribbons
}

\author{
E. Suárez Morell, R. Vergara, and M. Pacheco \\ Departamento de Física, Universidad Técnica Federico Santa María, Casilla 110-V, Valparaíso, Chile
}

L. Brey and Leonor Chico

Departamento de Teoría y Simulación de Materiales, Instituto de Ciencia de Materiales de Madrid (ICMM), Consejo Superior de Investigaciones Científicas (CSIC), C/ Sor Juana Inés de la Cruz 3, 28049 Madrid, Spain

(Received 7 February 2014; revised manuscript received 15 April 2014; published 6 May 2014)

\begin{abstract}
We study the band structure, density of states, and spatial localization of edge states in twisted bilayer graphene nanoribbons. We devise these ribbons by cutting a stripe of commensurate twisted bilayer graphene along a direction with a maximum number of zigzag edge atoms. Due to the spatially inhomogeneous interlayer coupling, edge states stemming from regions with $A B$ stacking are closer to the energy of the Dirac point, whereas those arising from $A A$-stacked edge states are split in energy due to the stronger interlayer coupling. As opposed to bulk bilayer graphene, for which states near the Dirac point are localized in $A A$-stacked regions, the interplay of edge and moiré localization produces a distinct spatial distribution of low-energy states in these ribbons.
\end{abstract}

DOI: 10.1103/PhysRevB.89.205405

PACS number(s): 73.22.-f

\section{INTRODUCTION}

The intriguing dependence of the electronic properties of twisted bilayer graphene (TBG) on the relative rotation angle (RRA) between layers has attracted many theoretical [1-13] and experimental works [14-17]. If the rotation angle between the two graphene layers is large, i.e., from $10^{\circ}$ to $30^{\circ}$ with respect to the perfect $A B$ stacking, the system behaves as if it were composed of two uncoupled layers [18], with a linear dispersion relation and the same Fermi velocity as monolayer graphene. Below a RRA of approximately $10^{\circ}$, the Fermi velocity starts to diminish, and around a RRA of about $1^{\circ}$ the bands are completely flat $[4,5]$. For these low rotation angles, a moiré pattern in the density of states develops, and distinct stacking regions can be observed [4,5,19]. Regions with $A A$ and $A B$ stacking can be distinguished, whereas others seem to be built of displaced graphene layers, called for this reason slip regions. For these low rotation angles, states near the Fermi level are mostly localized in the $A A$ region, with a strong localization, giving rise to the bright areas of the moire pattern $[4,5]$. The flattening of the bands with diminishing angle has been experimentally observed in angle-dependent Van Hove singularities revealed in density of states measurements by scanning tunneling spectroscopy $[10,14,20]$ and by an increase of the intensity of Raman modes measured in TBG samples [21,22].

There is not a controllable way to produce TBG with a given angle, although some growth methods are more prone to yield twisted bilayers [14,23]. Moreover, it was recently shown that graphene nanoribbons (GNRs) can be obtained by unzipping carbon nanotubes $[24,25]$. In this way, twisted bilayer nanoribbons have been obtained by unzipping chiral multiwalled nanotubes [26].

Chiral graphene nanoribbons have edges with a mixture of armchair and zigzag components. It has been theoretically established that all edges, with the exception of the pure armchair, present zero-energy localized states with a predominant

*eric.suarez@usm.cl weight at the edge atoms [27,28]. In fact, in minimal [27] edges, zero-energy states stem from the zigzag terminations. In pure zigzag nanoribbons, zero-energy states are strongly localized at these atoms [29,30]. In chiral geometries, edge states are also related to the presence of zigzag edge atoms, although they present remarkable size effects [28]. Indeed, physical properties of chiral GNRs and general edges can have a strong dependence on chirality [31,32].

Not only has the magnetic and electronic behavior of GNRs been reported to be chirality dependent [33-37] but also their elastic and thermal characteristics [38,39]. Edge states of chiral GNR have been experimentally evidenced [33]. Recent experimental works point towards edge chirality control by means of electrical [40] or optical [41] tools, opening a novel way to engineer the properties of these systems.

It is thus an interesting issue to study the effect of such edge states in twisted graphene, where moiré patterns show another way to confine electronic states. The properties of twisted bilayer graphene flakes have been addressed recently [41], but the interplay of these edge-localized states with the twisted geometry in extended systems such as GNRs remains to be explored.

In this work we study the band structure, density of states, and spatial localization of electrons in twisted bilayer nanoribbons by means of tight-binding calculations. We are interested in low-energy, edge-localized states; therefore, we analyze ribbons with minimal edges and a majority of zigzag atoms, which are responsible for the appearance of such edge states. We design the twisted nanoribbons by cutting a commensurate twisted bilayer graphene along a direction such that the edge contains the maximum number of zigzag atoms.

From our numerical results, we conclude that in the edge regions where top and bottom zigzag terminations are $A A$ stacked, inter-ribbon tunneling between the dispersionless zero-energy states creates bonding and antibonding combinations with energies hundreds of $\mathrm{meV}$ away from the energy of the Dirac point. On the other hand, in regions where the stacking between zigzag atoms at the edges is $A B$, the displacement of the position of the zigzag atoms in the top 
layer with respect to the bottom layer minimizes the hopping, so the low-energy states are almost unperturbed. As the twisting angle decreases, the size of the $A A$ - and $A B$-stacked regions increases, and likewise the number of zero-energy states located in the edge regions with $A B$-stacked zigzag atoms. This is in contrast with the bulk twisted bilayer, where zero-energy states merge in the $A A$ regions when the twisting angle decreases. We find that the density of states of twisted bilayer ribbons develops a zero-energy peak when the rotation angle decreases. Part of this peak stems from the bulk $A A$ regions, whereas another contribution is due to the $A B$-stacked edges with predominance of zigzag atoms.

The rest of the paper is organized in the following way: In Sec. II we describe the geometry of the twisted bilayer nanoribbons and introduce the tight-binding model employed to calculate the electronic properties. Section III is dedicated to a review of the band structure of monolayer chiral graphene nanoribbons and bilayer graphene nanoribbons with the most symmetric stacking, namely, $A A$ and $A B$. In Sec. IV we present and discuss the electronic structure and local density of states of twisted graphene nanoribbons. We finish with our conclusions in Sec. V.

\section{GEOMETRY AND MODEL}

\section{A. Geometry}

We build the unit cell of the twisted bilayer nanoribbon (TBNR) starting from a unit cell (UC) of a commensurate TBG $[18,19]$, which in turn is obtained by rotating a Bernal-stacked bilayer. The rotation axis passes through a $B$ site, which has an atom in a layer situated at the center of a hexagon of the other layer.

In order to choose commensurate $\mathrm{UCs}$, a point of the crystal with coordinates $\mathbf{r}=m \mathbf{a}_{1}+n \mathbf{a}_{2}$ ( $n, m$ integers) is rotated to an equivalent site $\mathbf{t}_{1}=n \mathbf{a}_{1}+m \mathbf{a}_{2}$. Here the graphene lattice vectors are given by $\mathbf{a}_{1}=\frac{a}{2}(\sqrt{3},-1)$ and $\mathbf{a}_{2}=\frac{a}{2}(\sqrt{3}, 1)$, and $a=2.46 \AA$ is the graphene lattice constant. The unit cell vectors of the bilayer twisted cell can be chosen as $\mathbf{t}_{1}=n \mathbf{a}_{1}+m \mathbf{a}_{2}$ and $\mathbf{t}_{2}=-m \mathbf{a}_{1}+(n+m) \mathbf{a}_{2}$. Bilayer unit cells built in this way are usually labeled by the indices $(n, m)$ $[4,5]$. The corresponding relative rotation angle between layers is given by $\cos \theta=\left(n^{2}+4 m n+m^{2}\right) / 2\left(n^{2}+m n+m^{2}\right)$. The repetition of a TBG unit cell with $n=m+1$ along either $\mathbf{t}_{1}$ or $\mathbf{t}_{2}$, yields a chiral ribbon with edges having a predominantly armchair component.

We are interested in ribbons with a majority of zigzag atoms, which give rise to edge-localized states. Therefore, due to the symmetry of the lattice, we choose the unit cell vector along the edge of the ribbon at $30^{\circ}$ with respect to $\mathbf{t}_{1}$ or $\mathbf{t}_{2}$. One possible way to achieve this is to choose the edge translation vector $\mathbf{T}$ perpendicular to $\mathbf{t}_{1}$, with $\mathbf{T}=2 \mathbf{t}_{2}-\mathbf{t}_{1}$ (see Fig. 1). As $\mathbf{t}_{1}$ and $\mathbf{t}_{2}$ form an angle of $60^{\circ}$, it can be seen that the periodic edge vector $\mathbf{T}$ of this ribbon has a length $\sqrt{3}\left|\mathbf{t}_{1}\right|$, and its coordinates can be written as $(2 m+n, n-m)$ in the basis $\mathbf{R}_{1}, \mathbf{R}_{2}$, depicted in Fig. 2(a). This basis is chosen so that both edge indices are positive and match the notation of Ref. [28].

We consider ribbons with minimal edges [27], i.e., those with a minimum number of edge nodes with coordination number 2. The width of the ribbon is given by an integer

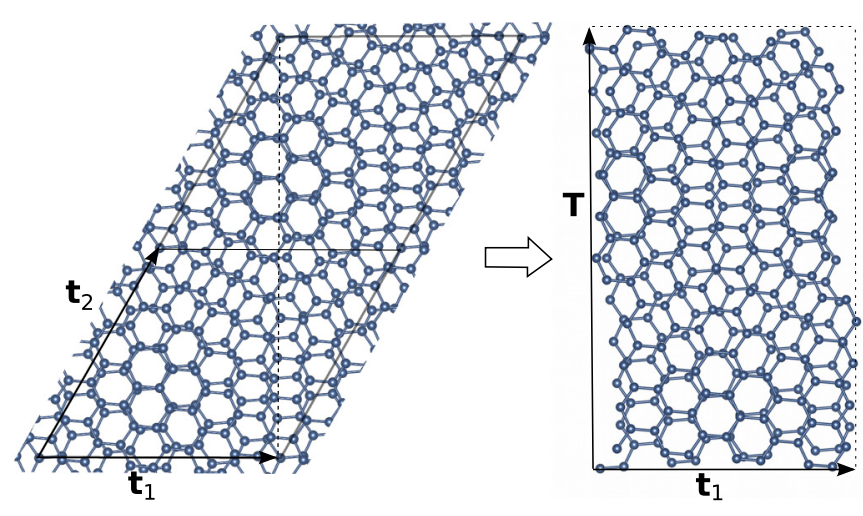

FIG. 1. (Color online) Construction of a TBNR unit cell following the procedure described in this work. In the left panel, two unit cells of a $(4,3)$ TBG are shown. The edge vector is chosen perpendicular to $\mathbf{t}_{1}$, i.e., along the dashed line. The right panel shows the unit cell for a $1(10,1)[(4,3)]$ twisted bilayer ribbon.

multiple $N$ of its width vector $\mathbf{t}_{1}$. From now on, we label the ribbons as $N(2 m+n, n-m)[(n, m)]$, where $(2 m+n, n-m)$ denotes the edge, $N$ the width, and $[(n, m)]$ identifies the moiré geometry. Even though this information is redundant for our particular construction, in general one should specify the edge and the moiré pattern, and even for our case we find it more convenient to give both.

For sufficiently small RRA, below $10^{\circ}$, distinct regions are observed in TBGs. There are areas with $A A$ stacking, others with $A B$ or $B A$ stacking, and there are also regions which seem to be obtained by a relative translation, called slip.

The UC of the ribbon constructed in this way contains one full $A A$ region in the lower part of the cell and the center of another $A A$ region is located in the upper part of the right edge, as can be seen in the right panel of Fig. 1. Moreover, there are zigzag edge atoms with either $A A$ or $A B$ stacking. This configuration will determine the analysis of the spatial localization of edge states.

\section{B. Tight-binding model}

We model the bilayer graphene nanoribbon within the tight-binding approximation including only the $p_{z}$ orbitals.

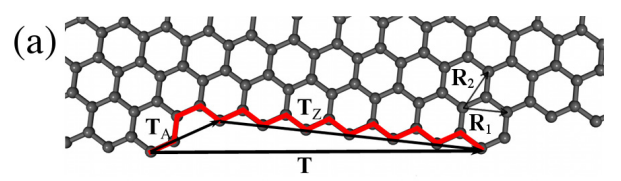

(b)

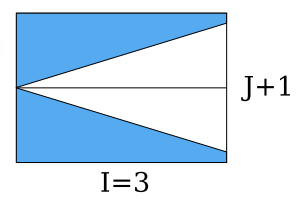

FIG. 2. (Color online) (a) Edge vector $\mathbf{T}(n, m)=(7,1)$ decomposed into its armchair and zigzag parts $\mathbf{T}_{Z}$ and $\mathbf{T}_{A}$, i.e., $(7,1)=$ $(6,0)+(1,1)$. The vectors $\mathbf{T}_{Z}$ and $\mathbf{T}_{A}$ are projections of $\mathbf{T}$ into the zigzag and armchair directions respectively. The primitive lattice vectors $\mathbf{R}_{1}$ and $\mathbf{R}_{2}$ are also shown so that $\mathbf{T}=n \mathbf{R}_{1}+m \mathbf{R}_{2}$. (b) Schematic band structure of a $(3 m, 0)$ edge, after folding $3 m$ times the $(1,0)$ zigzag edge band. The shaded areas represent the band continuum of states. 
Within each layer, we consider a fixed nearest-neighbor intralayer hopping parameter $\gamma_{0}=-3.16 \mathrm{eV}$. The distance between layers is set to $3.35 \AA$. For the layer-layer interaction we employ a distance-dependent hopping [3-5]. Thus, the Hamiltonian is given by $H=H_{1}+H_{2}+H_{12}$, where $H_{n}$ $(n=1,2)$ is the Hamiltonian for the individual layer $n$ and $H_{12}$ describes the interlayer coupling between the two layers:

$$
H_{12}=\sum_{i, j} \gamma_{1} e^{-\beta\left(r_{i j}-d\right)} c_{i}^{\dagger} c_{j}+\text { H.c. }
$$

where $\gamma_{1}=-0.39 \mathrm{eV}$ is the nearest-neighbor interlayer hopping parameter, $d$ is the interlayer distance, $r_{i j}$ is the distance between atom $i$ on layer 1 and atom $j$ on layer 2 , and $\beta=3$. This value of $\beta$ accurately reproduces the dispersion bands calculated within a density functional theory approach $[2,5,42]$. Every atom in a layer interacts with the atoms in the adjacent layer located inside a circle of radius $6 a_{\mathrm{CC}}$, where $a_{\mathrm{CC}}$ is the nearest-neighbor distance between carbon atoms, equal to $1.42 \AA$. This takes into account the complexity of the unit cell and at the same time breaks the electron-hole $(e-h)$ symmetry due to the fact that we are mixing the two sublattices. Notice that we employ the same interlayer Hamiltonian to model the interaction between the Bernal-stacked and the $A A$-stacked nanoribbons.

\section{PRELIMINARY THEORETICAL REMARKS}

The low-energy electronic properties of twisted bilayer ribbons depend on two main characteristics: their edge states and the presence of regions with $A A$ and $A B$ stacking. Therefore, we first review the most relevant features of chiral monolayer ribbons, as well as those of zigzag-terminated bilayer ribbons with the two most symmetric stackings, $A A$ and $A B$, with the aim of achieving a better understanding of the features of TBNRs.

\section{A. Band structure of chiral monolayer nanoribbons}

We first recall that minimal periodic graphene edges can be classified into four groups, depending on the position of the Dirac points in the one-dimensional (1D) Brillouin zone and on the wave vector span and degeneracies of the edge bands [28]. Armchair edges are the only case without edge states; all the rest do have zero-energy localized edge states due to the presence of zigzag edge atoms. This is the reason for our interest in ribbons with a predominance of zigzag-type edge atoms.

The type of zero-energy spectrum of a given periodic chiral edge with edge translation vector $\mathbf{T}=(p, q)$ (with $p \geqslant q$ ) is determined by its zigzag component $(p-q, 0)$ [28]. The edge spectrum is obtained by folding $p-q$ times the $(1,0)$ edge band, which corresponds to a pure zigzag edge. This leads to four possible cases, given by $p-q=I+3 J$, with $I=0,1,2,3$. The index $I$ determines the type of edge spectrum around the Dirac point and $J$ is related to the degeneracy of the edge bands. $I=0$ is the armchair case; it has also $J=0$.

Here we concentrate on twisted geometries with $n=$ $m+1$, as in Ref. [18]. The corresponding ribbons constructed following the prescription of the previous section have edges given by $(3 m+1,1)$, which are decomposed into a zigzag
$(3 m, 0)$ and an armchair $(1,1)$ part. This decomposition is illustrated for the $(7,1)$ edge in Fig. 2(a). Thus, all the electronic spectra from $N(3 m+1,1)[(m+1, m)]$ twisted ribbons belong to the same kind, namely, $I=3$, depicted in Fig. 2(b). They cover the whole Brillouin zone (BZ) with degeneracy $m$, so that the bilayer twisted ribbon has $4 m$ edge bands in total.

In a pure minimal zigzag edge [the $(1,0)]$, edge states are pinned at the Fermi level near the boundary of the first BZ, which corresponds to the $M$ point of the two-dimensional graphene BZ. We denote by $\bar{M}$ the projection of $M$ in the 1D BZ. For this $k$ value, edge states are maximally localized. For ribbons with other chiralities, the location of $\bar{M}$ changes due to the folding of the $(1,0)$ BZ. For the ribbons we study, with $(3 m+1,1)$ edges, it depends on whether $m$ is odd or even: $\bar{M}$ folds to the center of the BZ for $m$ odd and to the edge of the nanoribbon's first BZ zone otherwise.

Henceforth we denote the two special points of the nanoribbons as $\Gamma$ and $X$, which correspond to $k=0$, as is customary, and to the edge of the first BZ, respectively.

\section{B. Zigzag bilayer ribbons}

Bilayer zigzag nanoribbons with $A A$ and $A B$ stackings have been previously studied by tight-binding and densityfunctional methods [43-45]. Notice that for bilayer zigzag ribbons it is possible to have two different edge alignments; we choose the one with maximum overlap for the two zigzag ribbons, called $\beta$ stacking in previous works [43]. In zigzag and armchair ribbons, the width is commonly denoted by the number of dimers $N_{d}$ across its width. We follow here this widespread convention.

The band structures of $A A$ - and $A B$-stacked bilayer zigzag nanoribbons with $N_{d}=8$ and their corresponding geometries are shown in Fig. 3. For the sake of comparison, they have been calculated with the same Hamiltonian as the moire case,
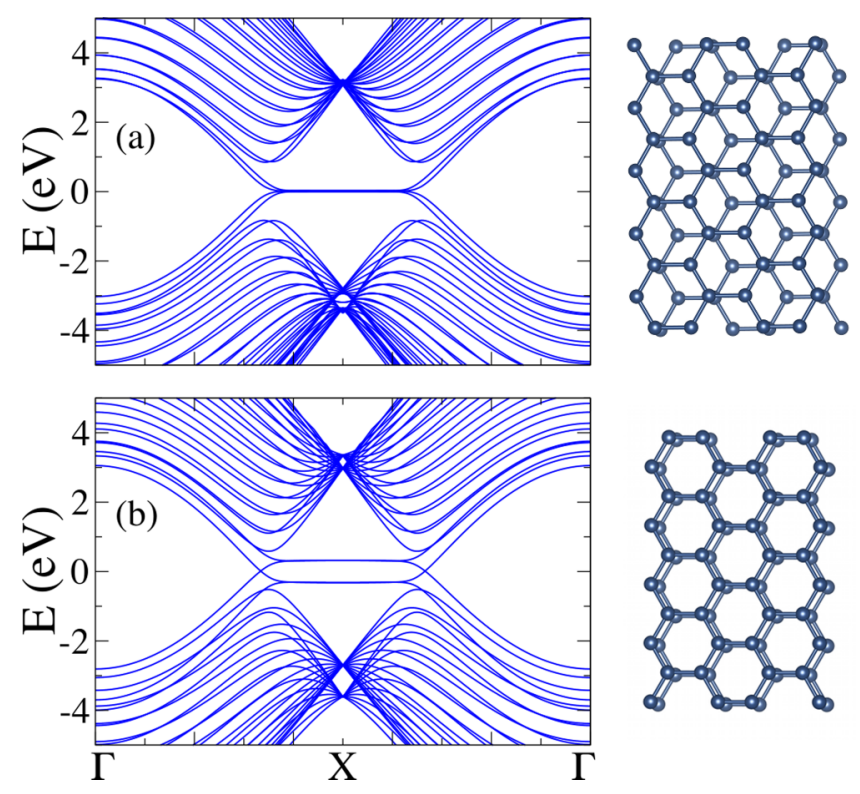

FIG. 3. (Color online) Band structures (left panels) and stacking geometries (right panels) of (a) $A B$ and (b) $A A$ bilayer graphene nanoribbons with widths $N_{d}=8$. 
presented in Sec. II B. As they are pure zigzag ribbons, four edge bands are expected for both bilayer ribbon cases. Indeed, for the $A B$ stacking there are four edge bands clearly identified in the figure, which are degenerate at the first Brillouin zone boundary at zero energy. This points to the lack of coupling for these bands. The bands for the $A A$-stacked ribbons show two Dirac-like band crossings shifted from $X$, and the edge flat bands are also split at $\pm \gamma_{1}$, due to the larger coupling. This splitting is independent of the width of the nanoribbon; it is due to the bonding-antibonding nature of the $A A$ states. Notice that zigzag bilayer nanoribbons do not have any gaps within the one-electron approximation.

\section{RESULTS AND DISCUSSION}

We start our discussion with the smallest TBNRs, the $N(4,1)[(2,1)]$. Having fewer atoms in the UC, their band structures are simpler, allowing for an easier analysis of their general behavior. These ribbons were generated from a twisted bilayer $(2,1)$ with a rotation angle $\theta=21.8^{\circ}$ and 28 atoms in the UC. The smallest corresponding TBNR has thus 56 atoms in the UC, the $1(4,1)[(2,1)]$.

The bilayer geometry and the two component monolayer ribbons are shown separately in Fig. 4, which also shows their corresponding band structures. The $(4,1)$ edge can be decomposed as $(3,0)+(1,1)$; indeed, in the right panels of Fig. 4 it can be seen that the edge has only one armchair $(1,1)$ step. This step is located at different parts of the superposed edges, so that they do not lie on top of each other. In addition, notice that the orientation of the $(1,1)$ steps is different in the two nanoribbons: in order to superimpose them with a perfect $A A$ stacking, a mirror reflection on a plane perpendicular to that of the ribbon is needed, in addition to a translation. Being related by a mirror reflection, the band structures of the two monolayer ribbons are equal.

As discussed in Sec. III, having a $(3,0)$ zigzag component, the $(4,1)$ edge has one zero-energy band. Thus, the monolayer ribbon should have two edge bands, and therefore the bilayer ribbon has four. These edge bands are split apart, as can be seen in the left panels of Fig. 4. This behavior is different from that of zigzag ribbons, due to the fact that the boundary condition for chiral edges mixes both sublattices. Such mixing leads to the coupling of edge states at opposite sides of each

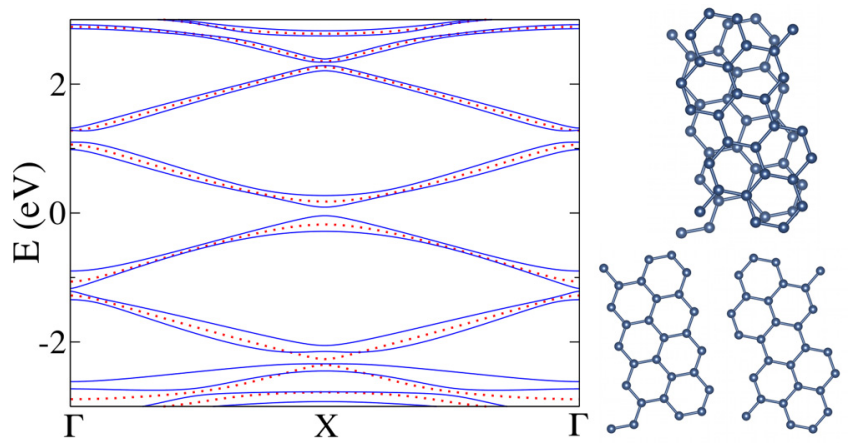

FIG. 4. (Color online) Band structures of the 1(4,1)[(2,1)] TBNR (solid lines) and of the corresponding monolayer ribbon (dotted lines). The geometry of the unit cell and its constituent monolayers are shown on the right.

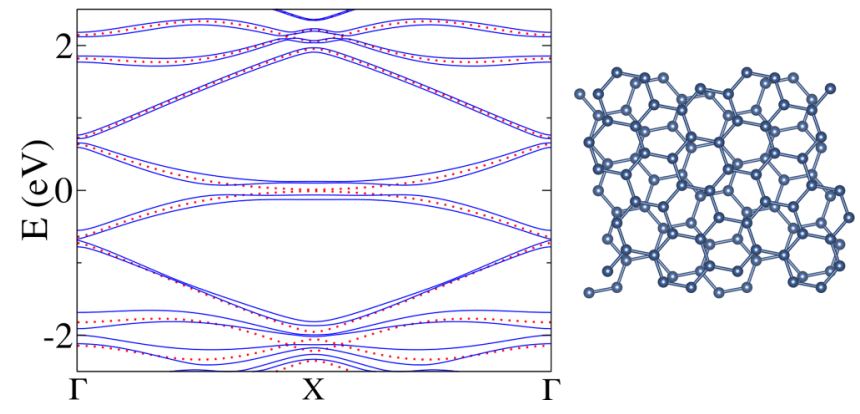

FIG. 5. (Color online) Left panel: Band structures of the $2(4,1)[(2,1)]$ TBNR (solid lines) and of the corresponding monolayer ribbon (dotted lines). Right panel: Unit cell of the $2(4,1)[(2,1)]$ TBNR.

monolayer ribbon, so that size effects produce the opening of a gap.

The gap between the two edge bands in the monolayer ribbon is wider than for the bilayer ribbon. Coupling between the two ribbons splits the bands, but as the ribbon is so narrow, it is difficult to distinguish whether the splitting is due to size effects or coupling.

In order to see the size effect we study a wider chiral ribbon with the same edge geometry, the $2(4,1)[(2,1)]$ TBNR, shown in Fig. 5. In the monolayer ribbon, the two edge bands are already decoupled: they touch at the edge of the BZ (as $m=1$, $\bar{M}$ is at the BZ boundary $X$ ) and their splitting at $\Gamma$ is also smaller. The gap at $\Gamma$ is greater than at $X$ because the states at $k=0$ are less localized. However, a gap of $\sim 0.2 \mathrm{eV}$ opens for the bilayer ribbon at $X$ due to the interlayer coupling. This gap can be related to the predominance of the $A A$ stacking in the edge zigzag atoms. As we saw for zigzag bilayer ribbons, $A A$ stacking leads to a bonding-antibonding splitting of the bands, which we also observe here.

We proceed now with a $1(13,1)[(5,4)]$ TBNR. This is built from a $(5,4)$ TBG, which has a RRA $\theta=7.34^{\circ}$ and 244 atoms in the unit cell. The corresponding ribbons have a $(13,1)$ edge, which decomposes as a $(12,0)+(1,1)$. Thus, the bilayer ribbon has 16 edge states. For smaller RRAs, as in this case, we can clearly distinguish the $A B$ and $A A$ zones in the UC (Fig. 6). For this ribbon $m=4$, so the $\bar{M}$ point is folded at $\Gamma$,
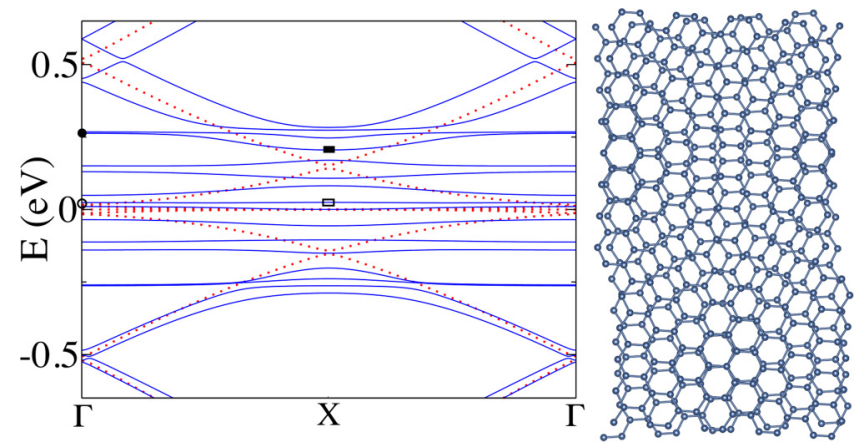

FIG. 6. (Color online) Left panel: Band structure of the $1(13,1)[(5,4)]$ TBNR (solid lines) and of the corresponding monolayer ribbon (dotted lines). Small circles and boxes indicate the $k$ points of the bands selected to show the local density of states in Fig. 7. Right panel: Unit cell of the 1(13,1)[(5,4)] bilayer ribbon. 
i.e., the edge bands are less dispersive near $k=0$. This ribbon, with $N=1$, is wide enough so as to identify some of the edge bands. The two edge bands closer to zero are barely split, so we can infer their $A B$ character.

In twisted bilayer graphene with a small rotation angle, the states with energies near that of the Dirac point are mostly localized in the $A A$-stacked regions [19], thus presenting a nonhomogeneous spatial distribution. The localization is quite strong and it is the reason for the remarkable moire pattern in TBGs. On the other hand, chiral bilayer ribbons present localized states at the edges, but their energies depend on the type of stacking, being almost degenerate at zero energy for the $A B$ and presenting a splitting $\sim\left|\gamma_{1}\right|$ for the $A A$ case. In addition, chiral edges also present a nonhomogeneous spatial distribution of their edge states, in contrast to those nanoribbons with pure zigzag edges.

The twisted bilayer ribbons studied here have a mixture of $A A$ and $A B$ sites at the edges. By their energy dependence, the less dispersive bands, which are closer to zero, are mainly localized in the $A B$ regions of the edges. Additionally, the splitting of states related to $A A$ sites should be $\sim\left|\gamma_{1}\right|$, assuming that half of the edge atoms are $A A$ and the other half $A B$.

With these ideas in mind, let us have a closer look at the bands of the 1(13,1)[(5,4)] TBNR shown in Fig. 6. There are four valence bands and four conduction bands with energies $\left|E_{b}\right|<0.2 \mathrm{eV}$ at $\Gamma$. These bands should be localized at the $A B$ sites of the edges, whereas the other four bands which are roughly split by $0.5\left|\gamma_{1}\right|$ from the energy of the Dirac point, should be mostly localized in the $A A$ edge region. The remaining bands penetrate into the ribbon, having an appreciable weight in the inner region. We verify this analysis by plotting in Fig. 7 the local density of states (LDOS) for four selected states of the $1(13,1)[(5,4)]$ TBNR, marked with circles and boxes in the band structure shown in Fig. 6. We have chosen two bands and two $k$ points in each band. One of the selected bands is very close to the energy of the Dirac point, while the other is in the energy range $0.2-0.25 \mathrm{eV}$. In order to see the effect of folding, the chosen $k$ points are $\Gamma$ (corresponding to $\bar{M}$ for this ribbon), and $X$, at the edge of the $1 \mathrm{D}$ first BZ.

The LDOS plot confirms our previous analysis. Panels (a) and (b) of Fig. 7 correspond to the band closest to the energy of the Dirac point, labeled with empty symbols; the other band, farther from $E=0$, is labeled with filled symbols. With respect to the wave vector, Figs. 7(a) and 7(c), marked with a circle, correspond to the $\Gamma(\bar{M})$ point; the boxes mark states at $X$, at the BZ boundary. As we expected, edge states of the lowest band are localized around the $A B$ region of the ribbon edges. This behavior is the same at both $k$ points; however, notice that the states at $\Gamma(\bar{M})$, which are more localized, are mainly located on one side of the ribbon, while the other states have an appreciable weight at both sides.

It is interesting what happens to the edge states coming from the other selected band around $E=0.2 \mathrm{eV}$, Figs. 7(c) and 7(d). Notice that the energy of the band at $\Gamma$ is slightly higher than that at $X$. The $\Gamma$ states are localized in the $A A$ region of the ribbon edge, and only on one side. The state at $X$ in Fig. 7(d) is more dispersive, because its energy is not quite enough to be fully at the $A A$ site: the state has an appreciable weight at both sides of the nanoribbon. Additionally, we would like to (a)

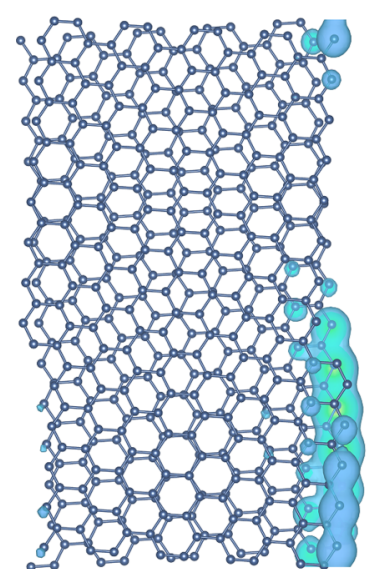

(c)

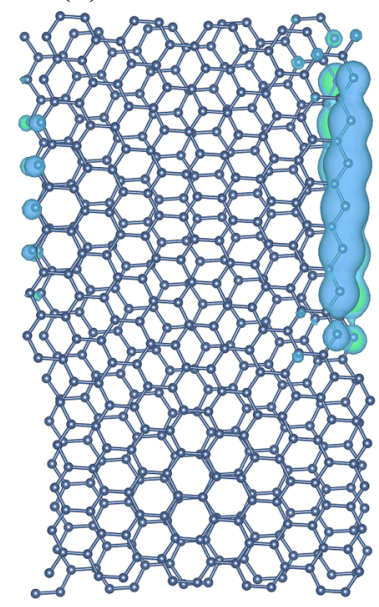

(b)

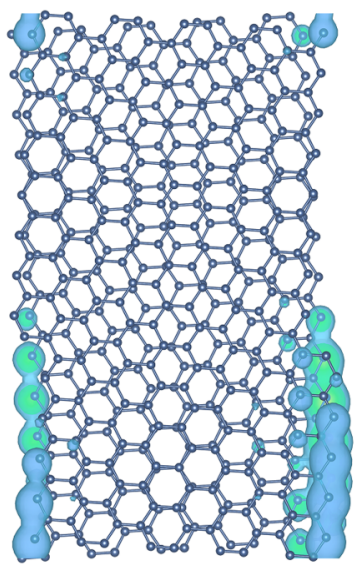

(d)

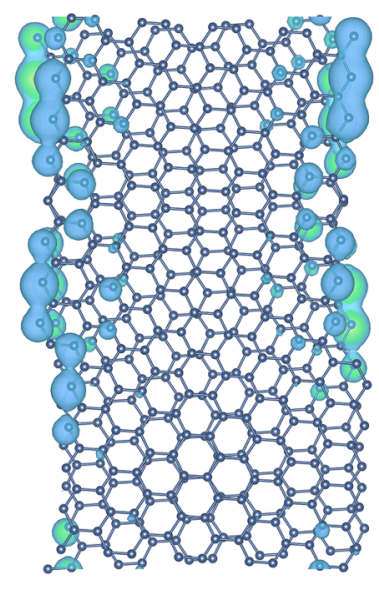

FIG. 7. (Color online) LDOS for four states belonging to bands near the Fermi level of a 1(13,1)[5,4] TBNR, as marked in Fig. 6. (a) Lowest energy state at $\Gamma$, marked with an empty circle; (b) lowest energy state at $X$, marked with an empty box; (c) highest energy state at $\Gamma$, marked with a filled circle; (d) highest energy state at $X$, marked with a filled box.

mention that we have checked that states farther apart from the energy of the Dirac point are fully extended throughout the ribbon.

A comment about other types of chiral edges is now in order. As we are interested in low-energy properties, relevant for transport properties, we have chosen edges with a maximum number of zigzag atoms, but our calculation method can be easily applied to any other structure. More importantly, we can also extend our conclusions to other kinds of chiral minimal edges: as low-energy states are related to the zigzag atoms, depending on whether they have an approximately $A B$ or $A A$ stacking, they will give rise to states closer to zero energy $(A B)$ or split apart by a quantity related to the interlayer coupling $(A A)$. The details of the final band structure will certainly depend on the particular stacking of the zigzag edge atoms, their proportion along the edge, their relative position, and size effects, but the mechanism to explain their localization and energy position is the same as for the ribbons studied in this paper, given above. 

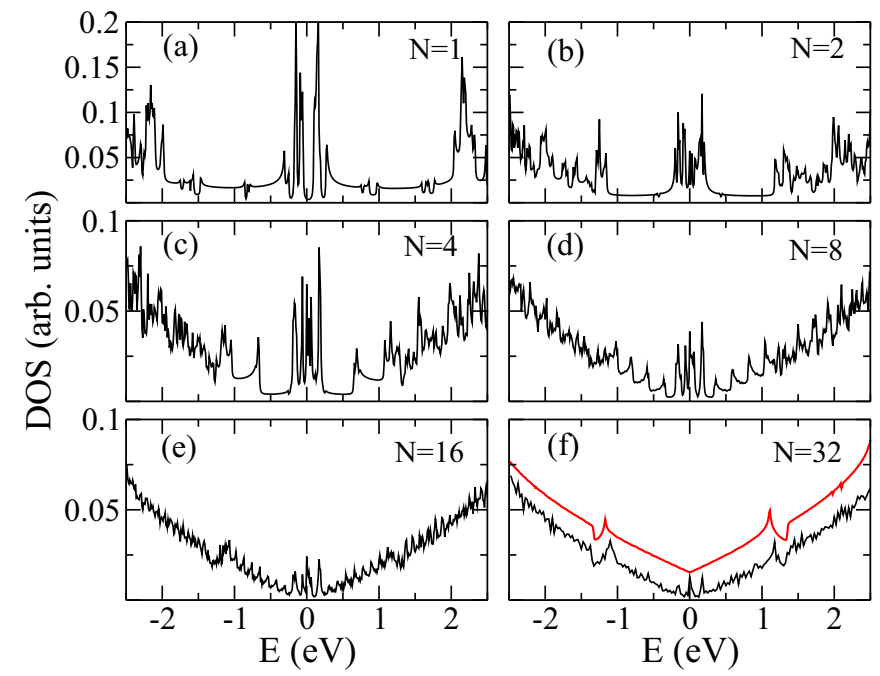

FIG. 8. (Color online) DOS for a series of $N(7,1)[(3,2)]:$ (a) $N=$ 1; (b) $N=2$; (c) $N=4$; (d) $N=8$; (e) $N=16$; (f) $N=32$. The bulk DOS for the $(3,2)$ twisted bilayer graphene is shown with a red line in (f), shifted upwards for comparison purposes.

\section{A. Density of states}

Finally, in Fig. 8 we have plotted the density of states (DOS) of an $N(7,1)[(3,2)]$ ribbon for six different widths, from $N=1$ to $N=32$. In panel (f) we show also the DOS of the corresponding $(3,2)$ twisted bilayer structure with the characteristic Van Hove singularities in the visible range.

The intensities of the zero-energy peaks decrease as the thickness of the nanoribbon increases, so that the DOS features change from clearly one dimensional [Fig. 8(a), $N=1$ ] to a more two-dimensional character [Fig. 8(f), $N=32$ ]. Although the DOSs of the ribbons evolve, as expected, to the bulk DOS as the ribbons gets wider, edge states persist around zero energy even for very wide ribbons. This shows the relevance of edge states even for very large structures. In fact, for small twist angles, for which the Fermi velocity tends to zero, an extra peak appears at $E=0$.

\section{SUMMARY AND CONCLUSIONS}

We have studied the band structure, DOS, and spatial localization of twisted bilayer graphene nanoribbons with edges of predominant zigzag character. We focus on a family of TBNRs with $(3 m+1,1)$ chiral edges, stemming from a simple geometrical construction from the customary unit cell employed for $(m+1, m)$ twisted bilayer graphene; however, these results can be easily generalized to other geometries. These $N(3 m+1)[(m+1, m)]$ TBNRs have $4 m$ edge states. We have found that the bands with energies $\left|E_{b}\right|<0.2 \mathrm{eV}$ are mainly localized in the $A B$ regions of the edges, while the edge bands farther from $E_{F}$ correspond to the $A A$ edge sites, following the moiré pattern of the bulk. Based on the characteristics of edge states of $A A$ - and $A B$-stacked bilayer nanoribbons and monolayer chiral ribbons, we have analyzed the properties of edge states in these twisted bilayer ribbons, explaining their energy dispersion and spatial localization. The different splittings observed in edge bands are due to the inhomogeneous interlayer coupling. For $A A$-stacked zigzag atoms, the coupling is larger and thus their corresponding edge states split apart; but edge states stemming from regions with $A B$ stacking, where the interlayer coupling is smaller, give rise to zero-energy bands. Due to the presence of edge states, the localization induced by the moiré pattern is strongly modified for twisted bilayer ribbons of nanometric size.

\section{ACKNOWLEDGMENTS}

L.C. acknowledges M. Pelc for helpful discussions. This work has been partially supported by MEC-Spain under Grant No. FIS2012-33521. E.S.M. acknowledges FONDECYT Grant No. 11130129. M.P. acknowledges FONDECYT Grant No. 1100672 and DGIP/USM Internal Grant No. 11.11.62.
[1] J. M. B. Lopes dos Santos, N. M. R. Peres, and A. H. Castro Neto, Phys. Rev. Lett. 99, 256802 (2007).

[2] S. Shallcross, S. Sharma, and O. A. Pankratov, Phys. Rev. Lett. 101, 056803 (2008).

[3] S. Shallcross, S. Sharma, E. Kandelaki, and O. A. Pankratov, Phys. Rev. B 81, 165105 (2010).

[4] G. Trambly de Laissardière, D. Mayou, and L. Magaud, Nano Lett. 10, 804 (2010).

[5] E. Suárez Morell, J. D. Correa, P. Vargas, M. Pacheco, and Z. Barticevic, Phys. Rev. B 82, 121407 (2010).

[6] E. J. Mele, Phys. Rev. B 81, 161405 (2010).

[7] R. Bistritzer and A. H. MacDonald, Proc. Natl. Acad. Sci. U.S.A. 108, 12233 (2011).

[8] J. M. B. Lopes dos Santos, N. M. R. Peres, and A. H. Castro Neto, Phys. Rev. B 86, 155449 (2012).

[9] P. San-Jose, J. González, and F. Guinea, Phys. Rev. Lett. 108, 216802 (2012).

[10] G. Trambly de Laissardière, D. Mayou, and L. Magaud, Phys. Rev. B 86, 125413 (2012).
[11] E. Suárez Morell, M. Pacheco, L. Chico, and L. Brey, Phys. Rev. B 87, 125414 (2013).

[12] T. Stauber, P. San-Jose, and L. Brey, New J. Phys. 15, 113050 (2013).

[13] J. D. Correa, M. Pacheco, and E. Suárez Morell, J. Mater. Sci. 49, 642 (2014).

[14] G. Li, A. Luican, J. M. B. Lopes dos Santos, A. H. Castro Neto, A. Reina, J. Kong, and E. Y. Andrei, Nat. Phys. 6, 109 (2010).

[15] A. Luican, G. Li, A. Reina, J. Kong, R. R. Nair, K. S. Novoselov, A. K. Geim, and E. Y. Andrei, Phys. Rev. Lett. 106, 126802 (2011).

[16] I. Brihuega, P. Mallet, H. González-Herrero, G. Trambly de Laissardière, M. M. Ugeda, L. Magaud, J. M. Gómez-Rodríguez, F. Ynduráin, and J.-Y. Veuillen, Phys. Rev. Lett. 109, 196802 (2012).

[17] L. Xian, S. Barraza-Lopez, and M. Y. Chou, Phys. Rev. B 84, 075425 (2011).

[18] E. Suárez Morell, P. Vargas, L. Chico, and L. Brey, Phys. Rev. B 84, 195421 (2011). 
[19] J. M. Campanera, G. Savini, I. Suarez-Martinez, and M. I. Heggie, Phys. Rev. B 75, 235449 (2007).

[20] W. Yan, M. Liu, R.-F. Dou, L. Meng, L. Feng, Z.-D. Chu, Y. Zhang, Z. Liu, J.-C. Nie, and L. He, Phys. Rev. Lett. 109, 126801 (2012).

[21] K. Sato, R. Saito, C. Cong, T. Yu, and M. S. Dresselhaus, Phys. Rev. B 86, 125414 (2012).

[22] K. Kim, S. Coh, L. Z. Tan, W. Regan, J. M. Yuk, E. Chatterjee, M. F. Crommie, M. L. Cohen, S. G. Louie, and A. Zettl, Phys. Rev. Lett. 108, 246103 (2012).

[23] F. Varchon, P. Mallet, L. Magaud, and J.-Y. Veuillen, Phys. Rev. B 77, 165415 (2008).

[24] D. V. Kosynkin, A. L. Higginbotham, A. Sinitski, J. R. Lomeda, A. Dimiev, B. K. Price, and J. M. Tour, Nature (London) 458, 872 (2009).

[25] L. Jiao, L. Zhang, X. Wang, G. Diankov, and H. Dai, Nature (London) 458, 877 (2009).

[26] L. Xie, H. Wang, C. Jin, X. Wang, L. Jiao, K. Suenaga, and H. Dai, J. Am. Chem. Soc. 133, 10394 (2011).

[27] A. R. Akhmerov and C. W. J. Beenakker, Phys. Rev. B 77, 085423 (2008).

[28] W. Jaskólski, A. Ayuela, M. Pelc, H. Santos, and L. Chico, Phys. Rev. B 83, 235424 (2011).

[29] K. Nakada, M. Fujita, G. Dresselhaus, and M. S. Dresselhaus, Phys. Rev. B 54, 17954 (1996).

[30] L. Brey and H. A. Fertig, Phys. Rev. B 73, 235411 (2006).

[31] V. Barone, O. Hod, and G. E. Scuseria, Nano Lett. 6, 2748 (2006).

[32] Y. Liu, A. Dobrinsky, and B. I. Yakobson, Phys. Rev. Lett. 105, 235502 (2010).
[33] C. Tao, L. Jiao, O. V. Yazyev, Y.-C. Chen, J. Feng, X. Zhang, R. B. Capaz, J. M. Tour, A. Zettl, S. G. Louie, H. Dai, and M. F. Crommie, Nat. Phys. 7, 616 (2011).

[34] O. V. Yazyev, R. B. Capaz, and S. G. Louie, Phys. Rev. B 84, 115406 (2011).

[35] M. Golor, T. C. Lang, and S. Wessel, Phys. Rev. B 87, 155441 (2013).

[36] L. Chico, H. Santos, M. C. Muñoz, and M. P. López-Sancho, Solid State Commun. 152, 1477 (2012).

[37] H. Santos, M. C. Muñoz, M. P. López-Sancho, and L. Chico, Phys. Rev. B 87, 235402 (2013).

[38] H. Zhao, K. Min, and N. R. Aluru, Nano Lett. 9, 3012 (2009).

[39] Z. Aksamija and I. Knezevic, Appl. Phys. Lett. 98, 141919 (2011).

[40] X. Jia, M. Hofmann, V. Meunier, B. G. Sumpter, J. CamposDelgado, J. M. Romo-Herrera, Y.-P. Son, H. Hsieh, A. Reina, J. Kong, M. Terrones, and M. S. Dresselhaus, Science 323, 1701 (2009).

[41] M. Begliarbekov, K.-I. Sasaki, O. Sul, E.-H. Yang, and S. Strauf, Nano Lett. 11, 4874 (2011).

[42] S. Latil, V. Meunier, and L. Henrard, Phys. Rev. B 76, 201402(R) (2007).

[43] B. Sahu, H. Min, A. H. MacDonald, and S. K. Banerjee, Phys. Rev. B 78, 045404 (2008).

[44] M. P. Lima, A. Fazzio, and A. J. R. da Silva, Phys. Rev. B 79, 153401 (2009)

[45] H. Santos, A. Ayuela, L. Chico, and E. Artacho, Phys. Rev. B 85, 245430 (2012). 